\title{
Synchronous Colonic Adenocarcinoma and Metastatic Lobular Carcinoma in a Colectomy Specimen: A Rare Finding
}

\author{
Nektarios Koufopoulos ${ }^{1}$, Christina Goudeli ${ }^{2}$, Eleni Pigadioti ${ }^{1}$, Dimitrios Balalis ${ }^{3}$, Dimitrios K. Manatakis ${ }^{4}$ \\ , Foteini Antoniadou ${ }^{1}$, Dimitris P. Korkolis ${ }^{3}$ \\ 1. Department of Pathology, "Saint Savvas” Cancer Hospital, Athens, GRC 2. Department of Gynecology, "Saint Savvas” \\ Cancer Hospital, Athens, GRC 3. Department of Surgery, "Saint Savvas” Cancer Hospital, Athens, GRC 4. Department of \\ Surgical Oncology, “Saint Savvas” Cancer Hospital, Athens, GRC
}

Corresponding author: Nektarios Koufopoulos, koufonektar@yahoo.com

\begin{abstract}
Invasive lobular carcinoma is the second-most-common subtype of invasive breast carcinoma. Its metastatic pattern is different compared to invasive carcinoma-no special type. It metastasizes more often to the gastrointestinal tract, peritoneum, pleura, and ovaries. The extrahepatic gastrointestinal tract metastases occur mostly in the stomach and small intestine and less often in the colon and rectum.

We present a case description of an 87-year-old woman admitted to our hospital with hematochezia, abdominal discomfort, fatigue, and weight loss. A colonoscopy revealed an exophytic tumor of the sigmoid colon. Metastatic disease was not found in imaging studies. A low anterior resection was performed. The pathologic examination revealed a collision tumor consisting of a poorly differentiated adenocarcinoma of the colon and metastatic lobular carcinoma. The diagnosis was challenging due to the lack of a previous history. Also, the diffuse architectural pattern and signet ring cells found may be in primary signet ring carcinoma of the colon as well as in carcinomas from other anatomical sites. Immunohistochemistry was helpful in making the diagnosis. A review of the literature revealed that this is the fourth case of metastatic breast carcinoma coexisting with colonic adenocarcinoma.
\end{abstract}

Categories: Pathology

Keywords: lobular carcinoma, metastasis, colon, colorectal carcinoma, gata-3, cdx-2, immunohistochemistry

\section{Introduction}

Invasive lobular carcinoma (ILC) is the second-most-common subtype of invasive carcinoma of the breast, accounting for up to $15 \%$ of cases. The classical variant displays multifocality, a diffuse architectural, the single-file linear pattern of tumor cells arranged in a concentric or targetoid pattern around normal ducts or structures, and intracellular mucin production either in the form of intracytoplasmic lumina or signet ring cells [1]. Several variants have been reported in the literature besides the classical ILC, including solid, signet ring cell, tubulolobular, alveolar, trabecular, pleomorphic, and a recently described variant with extracellular mucin production [2]. The latter variant shows both intra- and extracellular mucin production, a rare feature among breast carcinomas [3]. The ILC metastatic pattern is different compared to invasive ductal carcinoma, metastasizing more often to the gastrointestinal (GI) tract, peritoneum, pleura, and ovaries [4-5] whereas invasive carcinoma of no special type (NST) metastasizes more often to the lungs, liver, and brain [4]. Extrahepatic GI tract metastasis involves more often the stomach and the small intestine and, very rarely, the colon and rectum [6]. Other rare metastatic sites include the appendix [7], endometrium [8], gallbladder [9], kidney [10], orbit [11], pancreas [12], parotid gland [13], spleen [14], urinary bladder [15], uterine cervix [8], uterus [16], and vulva [17].

We herein describe a case of collision tumor in an 87-year-old woman consisting of a poorly differentiated colonic adenocarcinoma and a metastatic lobular carcinoma as an incidental finding in a colectomy specimen and a review of the literature.

\section{Case Presentation}

An 87-year-old patient with no previous history was admitted to our hospital due to hematochezia, abdominal discomfort, fatigue, and weight loss. Colonoscopy showed an exophytic tumor of the sigmoid colon. No evidence of metastatic disease was found on computed tomography (CT) scan or MRI. A low anterior resection was performed.

A pathologic evaluation of the resected specimen revealed a poorly differentiated adenocarcinoma of the colon with focal extracellular mucin production (Figure 1) and pericolic fat infiltration. There was a second neoplastic population found mainly near the serosa, consisting of smaller cells with a diffuse architectural pattern, some of them displaying intracytoplasmic lumina (Figure 2). In some areas, both neoplastic 


\section{Cureus}

populations were found in close proximity (Figure 3). Several signet ring cells were present. Fourteen lymph nodes were infiltrated, five by the colonic carcinoma and nine by the second type of neoplastic cells (Figure 4). Due to the fact that there was no other neoplasm in her history, the second neoplastic population was assumed to be a minor component of primary colonic carcinoma with signet ring morphology. The differential diagnosis also included ILC and other metastatic carcinomas with a diffuse architectural pattern.

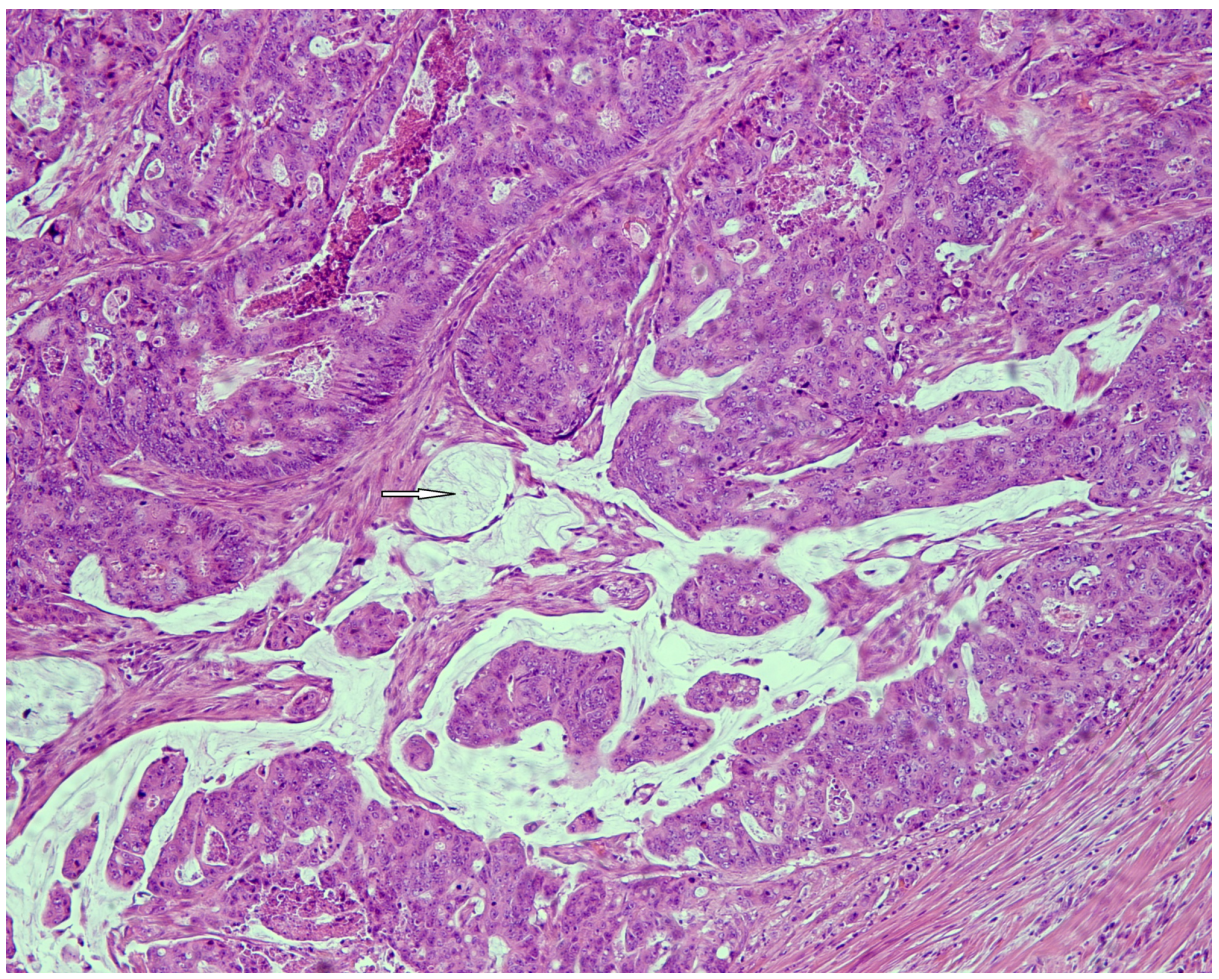

FIGURE 1: Poorly differentiated colonic adenocarcinoma displaying extracellular mucin production (white arrow) (HE x 100).

Abbreviation: HE: hematoxylin and eosin.

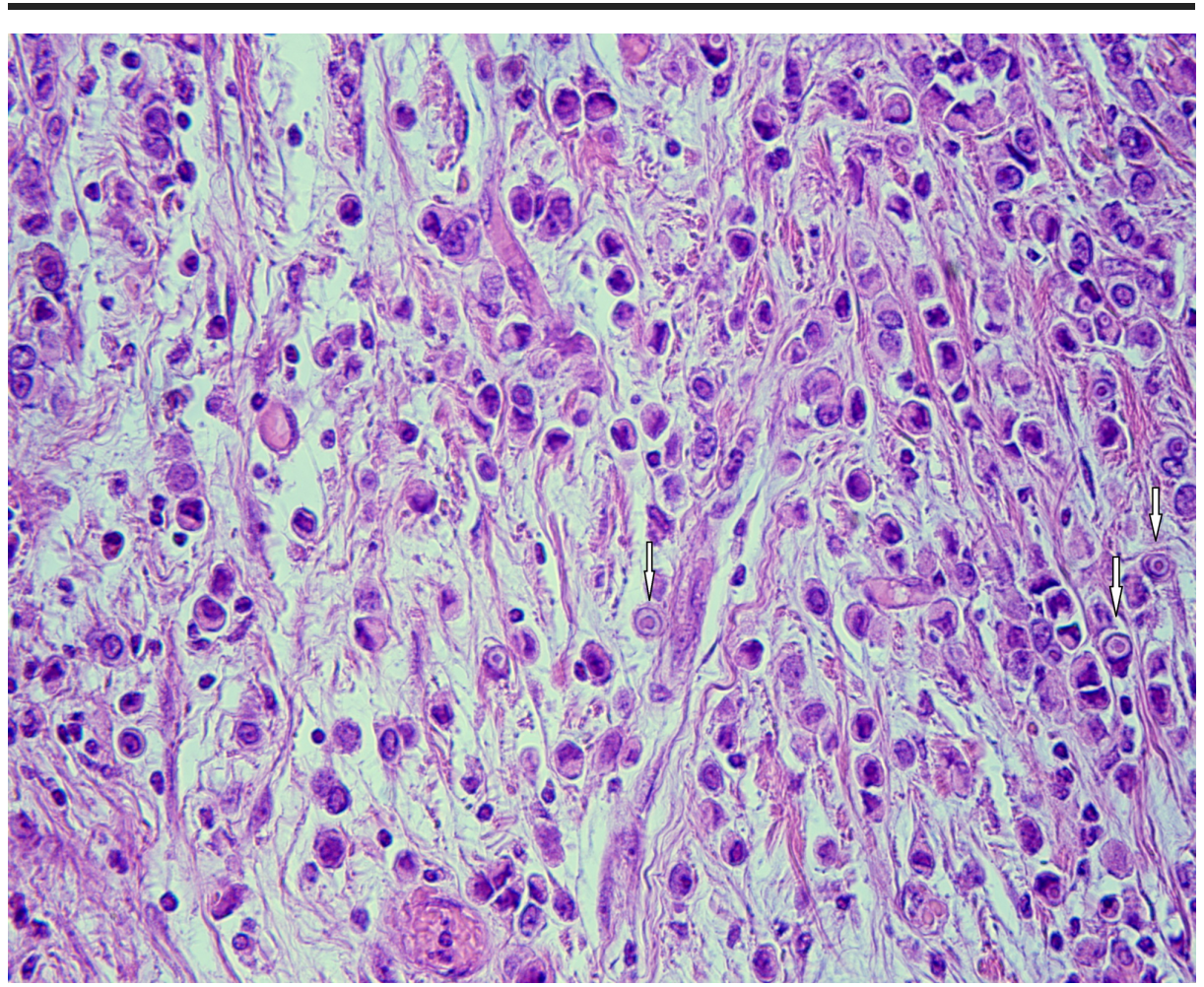




\section{Cureus}

FIGURE 2: Diffuse type carcinoma cells display eccentric nuclei and intracytoplasmic lumina (white arrows) at high magnification (HE x 400). Abbreviation: HE: hematoxylin and eosin.

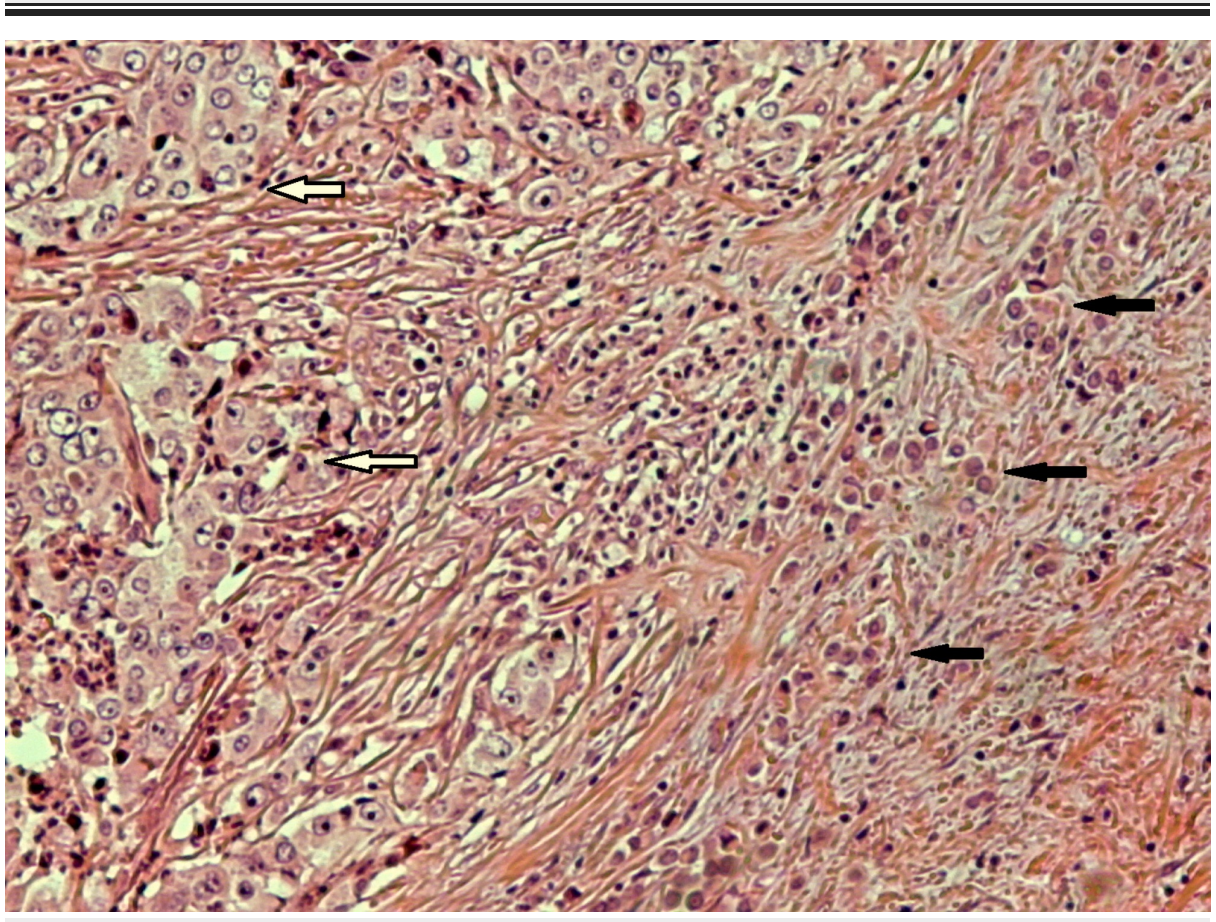

FIGURE 3: Nests of poorly differentiated colonic carcinoma (white arrows) in close proximity to smaller neoplastic cells (black arrows) with a diffuse architectural pattern (HE x 100).

Abbreviation: HE: hematoxylin and eosin.

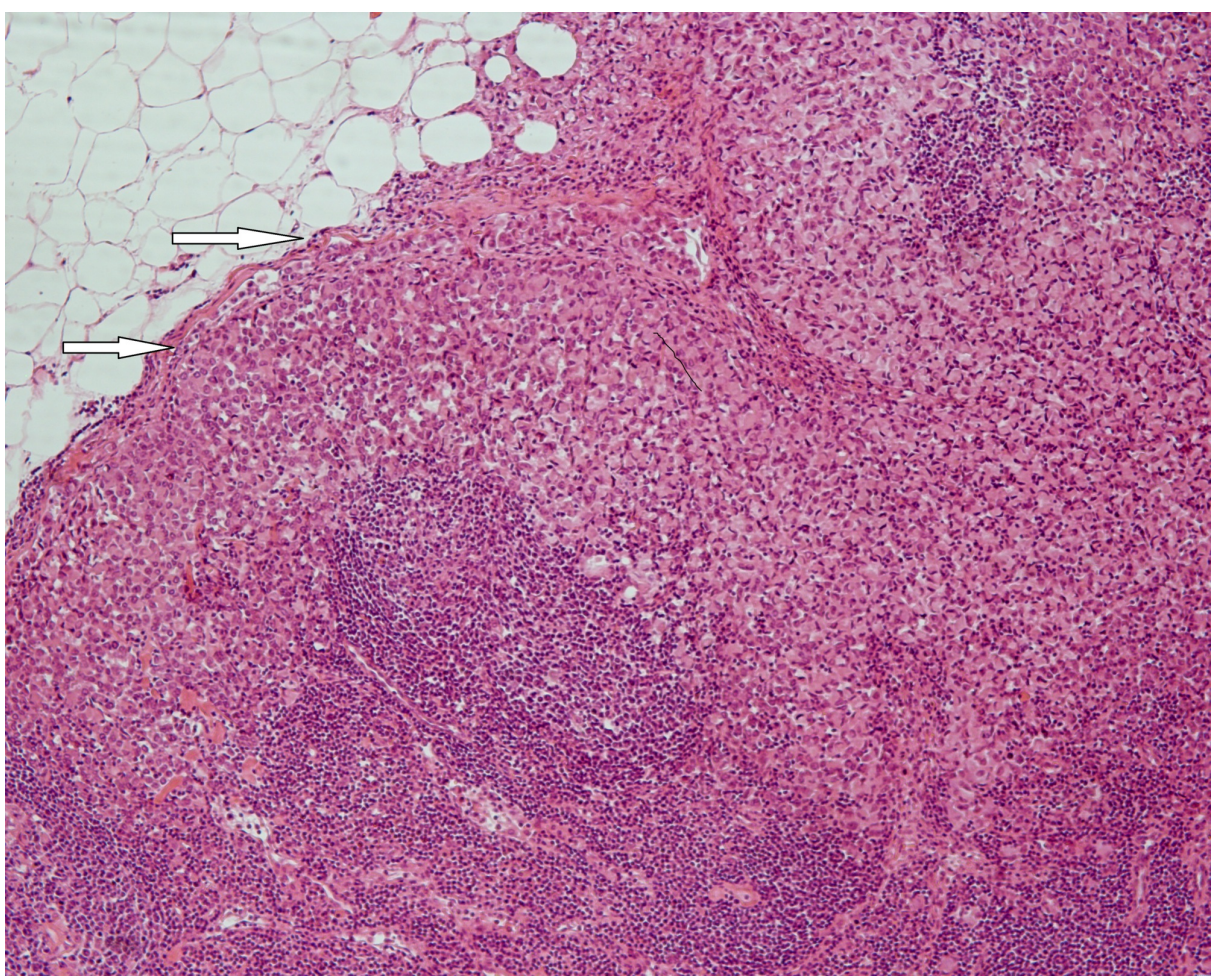




\section{Cureus}

FIGURE 4: Lymph node infiltration by diffuse type carcinoma cells (white arrows) (HE x 100).

Abbreviation: HE: hematoxylin and eosin.

The immunohistochemical study was negative for CK-20 and CDX-2 in the diffuse type carcinoma cells and positive for them in the colonic adenocarcinoma (Figure 5). CK-7 was negative in both. Additional stainings for GATA-3 (Figure 6), mammaglogin, estrogen receptor (ER)/progesterone receptor (PR) were performed, which were negative in the colonic adenocarcinoma and positive in the diffuse type carcinoma. E-cadherin was negative in the diffuse type carcinoma and positive in the colonic adenocarcinoma. Chromogranin and synaptophysin were negative in both. The diagnosis of a poorly differentiated colonic carcinoma with focal extracellular mucin production co-existing with a carcinoma consistent with metastatic ILC was made.

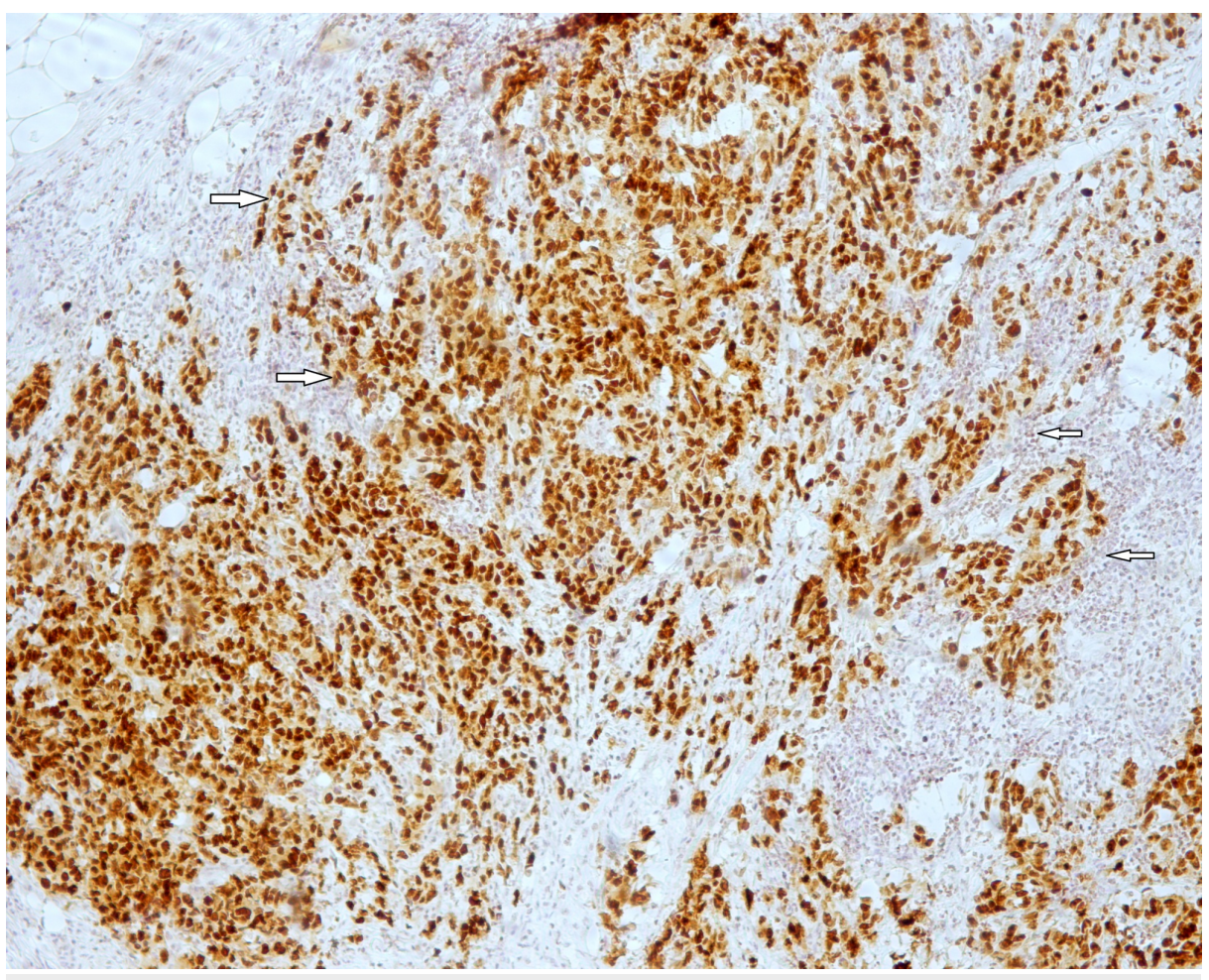

FIGURE 5: CDX-2 is diffusely positive in colonic carcinoma cells (white arrows) (CDX-2 x 100). 


\section{Cureus}

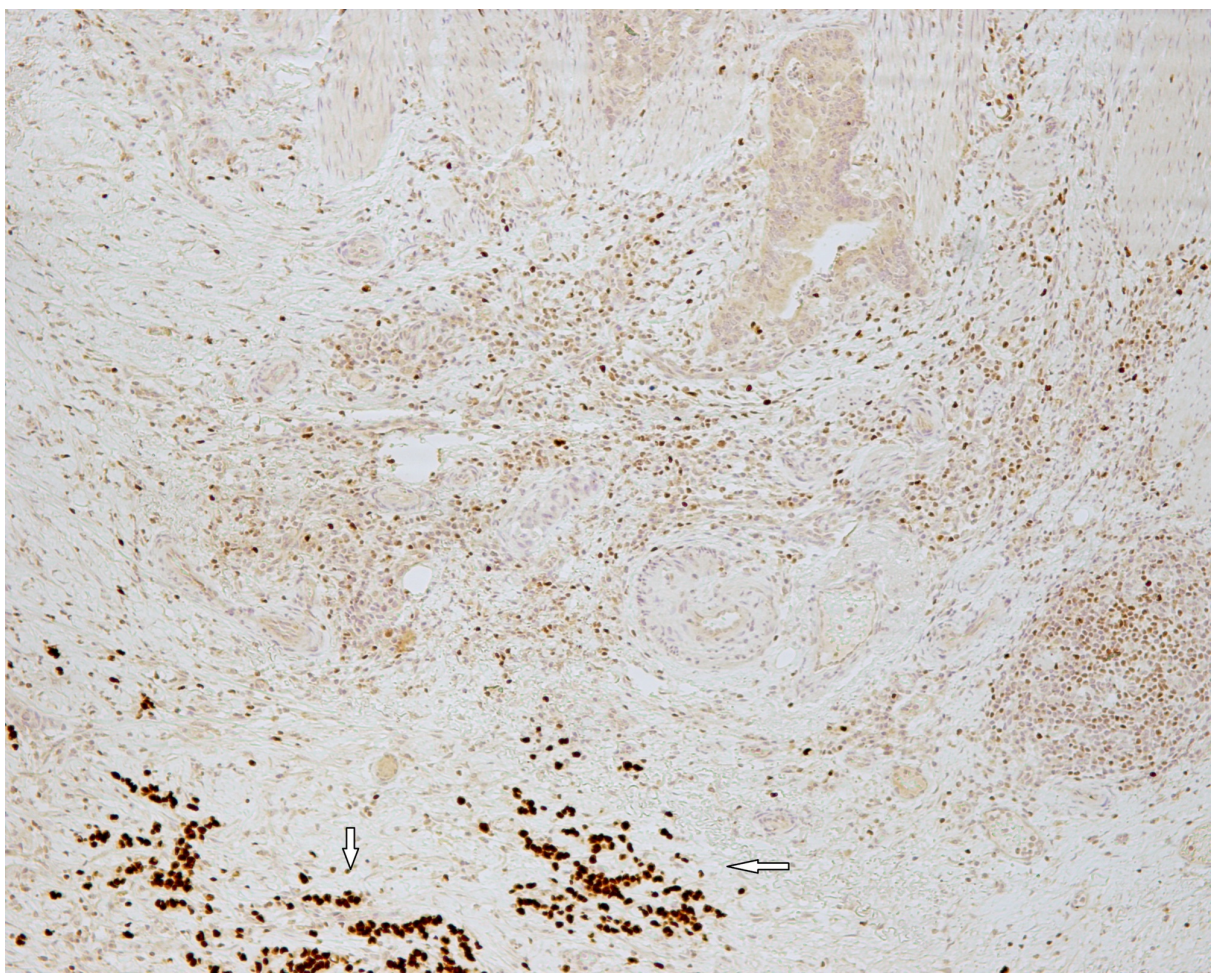

FIGURE 6: Diffuse type carcinoma cells were positive for GATA-3 (white arrows) while colonic adenocarcinoma cells were negative (GATA-3 $x$ 100).

Imaging studies did not reveal any primary breast tumor. Due to her advanced age, the multidisciplinary tumor board decided to treat her with hormonal therapy. The patient refused further treatment and was lost to follow up.

\section{Discussion}

Breast carcinoma is the most frequent cancer in women, accounting for approximately one-third of cancers, and is a significant cause of morbidity and mortality [18]. GI tract metastases usually occur four to five years after primary breast cancer diagnosis but sometimes 20 or even 30 years later. Occasionally, their presentation is concurrent but, rarely, they may be the first clinical manifestation of breast cancer $[4,19]$. The rate of GI tract involvement in metastatic breast cancer is around $1 \%$ and is found in up to $12 \%$ of autopsies [18].

The metastatic pattern of ILC is different from that of an NST carcinoma of the breast. The reason for this different metastatic pattern has been suggested to be the loss of E-cadherin [18]. ILC metastasizes more commonly to the GI tract than NST carcinoma of the breast with an incidence of $4.5 \%$ and $0.2 \%$, respectively [18] and is usually associated with disseminated disease [4].

Clinical diagnosis is difficult because colonic metastases may present as a mass mimicking a primary GI tumor, other benign GI tract tumors, or as Crohn's disease [18,20]. The histological diagnosis may be difficult as well. A previous history of breast malignancy can be very helpful in establishing the correct diagnosis. It has been suggested that for any woman presenting with new GI complaints and a history of breast carcinoma, metastasis must be considered [18]. Attention to histological detail paired with the previous history will usually suffice for diagnosis. In difficult cases, immunohistochemistry will provide the solution as breast carcinoma tumor cells are positive for CK-7, GATA-3, mammaglobin, GCDFP-15, and estrogen and progesterone receptors. In our case, despite the fact that CK-7 was negative, the diagnosis of metastatic ILC was made due to the fact that markers considered more specific for mammary carcinoma (GATA-3, mammaglobin, and GCDFP-15) were positive.

Our literature review has revealed three cases of breast carcinoma metastasis to the colon coexisting with colonic adenocarcinoma [4-5,20]. Two of these involved ILC and the third was NST carcinoma. In the first case, the metastatic ILC was undiagnosed until colorectal resection [4] whereas the other two cases had a known history of ILC [5,20]. The clinicopathological features of these cases are presented in Table 1. Interestingly, the patient with colonic metastasis by NST carcinoma had a history of ILC 30 years prior [20]. 


\section{Cureus}

The lymph nodes were infiltrated by both colorectal and breast carcinoma in the two cases involving ILC [4-

5]. As in our case, the primary tumor was not located by further imaging studies in one of the cases [4].

\begin{tabular}{|c|c|c|c|c|c|c|c|}
\hline Case & Year & Age & Timing & Breast Ca & Colon Ca & Adj. Therapy & Outcome (mo) \\
\hline $1^{4}$ & 2013 & 78 & 9 years & ILC * & CRC G2 & Horm+Chemo & 48 ANED \\
\hline $2^{20}$ & 2014 & 83 & Conc. & NST G2 & CRC G2 & Horm & 18 ANED \\
\hline $3^{5}$ & 2015 & 80 & Conc. & ILC G2 & CRC G2 & Horm & 4 ANED \\
\hline Present case & 2017 & 87 & Conc. & ILC G2 & CRC G3 & ** & ${ }^{\star \star \star *}$ \\
\hline
\end{tabular}

\section{TABLE 1: Clinicopathological features in cases of breast carcinoma metastasis to the colon co- existing with colonic adenocarcinoma.}

Abbreviations: Adj. Therapy: adjuvant therapy; ANED: alive no evidence of disease; Ca: cancer; Chemo: chemotherapy; Conc: concomitant; CRC: colorectal carcinoma; G: grade; Horm: hormonal treatment; ILC: invasive lobular carcinoma; mo: months; NST: no special type carcinoma.

\section{${ }^{*}$ Grade is not mentioned}

${ }^{* *}$ Patient refused treatment

***Patient was lost to follow up

There is no consensus regarding the treatment of metastatic breast cancer with intestinal involvement due to its rarity. Systemic treatment with surgery or surgery alone has been suggested to have a favorable outcome in some reports [20].

\section{Conclusions}

While breast carcinoma colorectal metastasis is a rare event, primary colonic and metastatic breast carcinoma coexistence is extremely rare. The precise diagnosis of metastatic breast carcinoma in a colectomy specimen may be very challenging, especially in cases lacking a previous history. In such cases, immunohistochemistry usually provides a diagnostic solution. Systemic treatment has been suggested to have a favorable outcome in some reports.

\section{Additional Information \\ Disclosures}

Human subjects: Consent was obtained by all participants in this study. Conflicts of interest: In compliance with the ICMJE uniform disclosure form, all authors declare the following: Payment/services info: All authors have declared that no financial support was received from any organization for the submitted work. Financial relationships: All authors have declared that they have no financial relationships at present or within the previous three years with any organizations that might have an interest in the submitted work. Other relationships: All authors have declared that there are no other relationships or activities that could appear to have influenced the submitted work.

\section{References}

1. Reed AEM, Kutasovic JR, Lakhani SR, Simpson PT: Invasive lobular carcinoma of the breast: Morphology, biomarkers and 'omics. Breast Cancer Res. 2015, 17:12. 10.1186/s13058-015-0519-x

2. Cserni G, Floris G, Koufopoulos N, et al.: Invasive lobular carcinoma with extracellular mucin production-a novel pattern of lobular carcinomas of the breast. Clinico-pathological description of eight cases. Virchows Arch. 2017, 471:3-12. 10.1007/s00428-017-2147-6

3. Koufopoulos N, Goudeli C, Syrios J, Filopoulos E, Khaldi L: Mucinous cystadenocarcinoma of the breast: the challenge of diagnosing a rare entity. Rare Tumors. 2017, 9:98-100. 10.4081/rt.2017.7016

4. Miller T, Ross C, Al-Rawi H, Taylor B, Al-Jafari M: Unexpected diagnosis of both adenocarcinoma of the colon and metastatic lobular carcinoma of the breast in the gastrointestinal tract. Case Rep Pathol. 2013, 2013:1-3. 10.1155/2013/153180

5. Mroz A, Kiedrowski M: An unusual case of colonic adenocarcinoma development in the region of disseminating lobular breast carcinoma infiltration: Diagnostic approach and review of the literature. Int J Clin Exp Pathol. 2015, 8:7470-7474.

6. Cervi G, Vettoretto N, Vinco A, Cervi E, Villanacci V, Grigolato P, Giulini SM: Rectal localization of metastatic lobular breast cancer. Dis Colon Rectum. 2001, 44:453-455. 10.1007/BF02234749

7. Dirksen JL, Souder MG, Burick AJ: Metastatic breast carcinoma presenting as perforated appendicitis. Breast 
Care. 2010, 5:409-410. 10.1159/000322656

8. Razia S, Nakayama K, Tsukao M, et al.: Metastasis of breast cancer to an endometrial polyp, the cervix and a leiomyoma: a case report and review of the literature. Oncol Lett. 2017, 14:4585-4592. 10.3892/ol.2017.6822

9. Markelov A, Taheri H, Vunnamadala K, Ibrahim G: Biliary dyskinesia as a rare presentation of metastatic breast carcinoma of the gallbladder: a case report. Case Rep Pathol. 2011, 2011:1-3. 10.1155/2011/806570

10. Al-Jarrah A, Taranikanti V, Sawhney S, Furrukh M, Al-Hosni M, Saparamadu PAM, De Silva MVC: Metastatic invasive lobular carcinoma of the breast masquerading as a primary renal malignancy. Sultan Qaboos Univ Med J. 2013, 13:453-455. 10.12816/0003271

11. Gupta S, Bhatt VR, Varma S: Unilateral orbital pain and eyelid swelling in a 46-year-old woman: orbital metastasis of occult invasive lobular carcinoma of breast masquerading orbital pseudotumour. BMJ Case Rep. 2011, 2011:1220103580. 10.1136/bcr.12.2010.3580

12. Molino C, Mocerino C, Braucci A, et al.: Pancreatic solitary and synchronous metastasis from breast cancer: a case report and systematic review of controversies in diagnosis and treatment. World J Surg Oncol. 2014, 12:2. 10.1186/1477-7819-12-2

13. Ando K, Masumoto N, Sakamoto M, et al.: Parotid gland metastasis of breast cancer: case report and review of the literature. Breast Care. 2011, 6:471-473. 10.1159/000335222

14. Groisman GM: Lobular carcinoma of the breast metastatic to the spleen and accessory spleen: report of a case. Case Rep Pathol. 2016, 2016:5160180. 10.1155/2016/5160180

15. Nieder C, Pawinski A: A case of recurrent breast cancer with solitary metastasis to the urinary bladder . Case Rep Oncol Med. 2014, 2014:1-3. 10.1155/2014/931546

16. Komeda S, Furukawa N, Kasai T, Washida A, Kobayashi H: Uterine metastasis of lobular breast cancer during adjuvant letrozole therapy. J Obstet Gynaecol (Lahore). 2013, 33:100-101. 10.3109/01443615.2012.721407

17. Papaioannou N, Zervoudis S, Grammatikakis I, Peitsidis P, Palvakis K, Youssef TF: Metastatic lobular carcinoma of the breast to the vulva: a case report and review of the literature. J Egypt Natl Canc Inst. 2010, 22:57-60.

18. Mistrangelo M, Cassoni P, Mistrangelo M, et al.: Obstructive colon metastases from lobular breast cancer: report of a case and review of the literature. Tumori. 2011, 97:800-804. 10.1700/1018.11099

19. Clavien PA, Laffer U, Torhost J, Harder F: Gastro-intestinal metastases as first clinical manifestation of the dissemination of a breast cancer. Eur J Surg Oncol. 1990, 16:121-126.

20. Jafferbhoy S, Paterson H, Fineron P: Synchronous gist, colon and breast adenocarcinoma with double colonic polyp metastases. Int J Surg Case Rep. 2014, 5:523-526. 10.1016/j.ijscr.2014.04.020 